\title{
Programa de mantenimiento con metadona (PMM) en prisión: Cambios sociales y sanitarios
}

\author{
Arroyo, A.*; Marrón, T.*; Coronas, D.**; Leal, M.J.***; Solé, C.****; Laliaga, A.**** \\ * Médico Forense Hospitalet de Llobregat. \\ * Servei Catalá de la Salut. \\ *** Centro Penitenciario de Jóvenes Barcelona. \\ *** Centro Penitenciario Brians Barcelona \\ Correspondencia: \\ Dra. Amparo Arroyo Fernández Passeig Terraplé $n^{\circ} 6,1^{\circ}-2^{\mathrm{a}}$. Molins de Rei. Barcelona.
}

\section{Resumen}

Introducción: Los PMM ayudan al drogodependiente que no ha tenido éxito en programas libres de drogas a adoptar una conducta social adecuada, disminuir los problemas legales y mejorar la salud.

Objetivos: Valorar los beneficios del tratamiento con metadona en heroinómanos, en comparación con las circunstancias anteriores a su inclusión en el programa. Valorar el consumo simultáneo de otras drogas y el consumo de metadona ilegal.

Pacientes y Métodos: Se diseñó un estudio pre-experimental con un solo grupo pre-post intervención. Se realizó una única entrevista y se recogió información sobre el estado de los sujetos antes y después de recibir el tratamiento. La muestra fue de 62 internos del Centro Penitenciario de Brians (Barcelona). Las variables a considerar fueron. trabajo, nivel socioeconómico, autoestima y autocontrol, problemática legal, tratamiento psiquiátrico, intentos de suicidio, hábito de compartir jeringuillas, prostitución, hetero y auto-agresividad e historia de drogadicción. El tratamiento estadístico aplicado fue comparación de proporciones para variables categóricas, test de Mc Nemar y Test de Friedman para variables ordinales.

Resultados: Durante la inclusión en el programa mejoraron de forma significativa las variables consideradas y disminuyó el consumo de cocaína y cannabis, aumentó el consumo de alcohol, nicotina, benzodiacepinas y drogas de diseño.

Discusión: Se comparan los resultados con otros estudios. En conclusión los PMM representan una estrategia terapéutica válida dentro y fuera de centros penitenciarios. Constituyen una alternativa eficaz para la consecución de objetivos de salud, disminuyen conductas de riesgo para VIH y otras patologías asociadas y modifican positivamente aspectos legales y sociales.

Palabras Clave: metadona, prisión, cambios sociosanitarios, drogodependencia.

\section{Summary}

Maintenance methadone programs help addicts who have previously failed in free- drugs programs to have better social behaviour, decreased criminality and legal problems and improved health.

Aims: To assess the benefits of methadone treatment in opiate addicts individuals, before and after being included in the program. To assess the effects of the simultaneous consumption of other drugs and illegal methadone.

Patients and Methods: A pre-experimental study was designed with a pre-post intervention group. A single interview was performed and information about the situation of patients before and after the methadone treatment was obtained. The patients were a group of 62 prisoners from the Brians Penitentiary Center ( Barcelona). Variables to be considered include: labour activity, social and economic level, self control and self esteem, legal problems, psychiatric treatment, suicide attempts, sharing of injecting equipment, prostitution, irritability and drug addiction background. The statistics methods applied were nonparametric tests, comparason of proportions for categorical variables, Mc Nemar and Friedman tests for ordinal variables.

Results: social and self esteem improved during inclusion of addicts in the treatment program. Cocaine and cannabis consumption diminished significantly. however Alcohol, nicotine, benzodiazepines and designer drug consumption increased during the length of the program.

Discussion: Results are compared with those stablished by other authors and published.. The conclusion is that methadone maintenance programs are a valid strategy in and out of penitentiary centers, diminish risk behaviour for HIV , hepatitis and other derived pathologies and modify social and legal aspects.

Key words: Methadone, prisson, sociosanitary changes, drugdependence.

\section{INTRODUCCIÓN}

a finalidad de los Planes de Mantenimiento con Metadona (PMM) consiste en ayudar al drogodependiente que no ha tenido éxito en los progra- mas de desintoxicación sin drogas a adoptar una conducta adecuada en la familia y sociedad, disminuir los problemas legales y mejorar la salud.

La metadona es un opiáceo de acción prolongada, derivado de la 3,3-difenilpropilamina que posee casi 


\begin{tabular}{|c|c|c|c|c|c|c|c|}
\hline \multicolumn{8}{|c|}{ TABLA $N^{\circ}$ 1. Edad media de inicio en el consumo de tóxicos } \\
\hline & ALCOHOL & NICOTINA & CANNABIS & HEROÍNA & COCAÍNA & BZD & D.DISEÑO \\
\hline EDAD MEDIA (años) & 12.8 & 12.7 & 13.4 & 16.7 & 18.2 & 16.3 & 16.1 \\
\hline RANGO & 7 y 21 & 7 y 19 & 9 у 26 & 11 y 31 & 12 y 31 & 9 y 24 & 11 y 36 \\
\hline
\end{tabular}

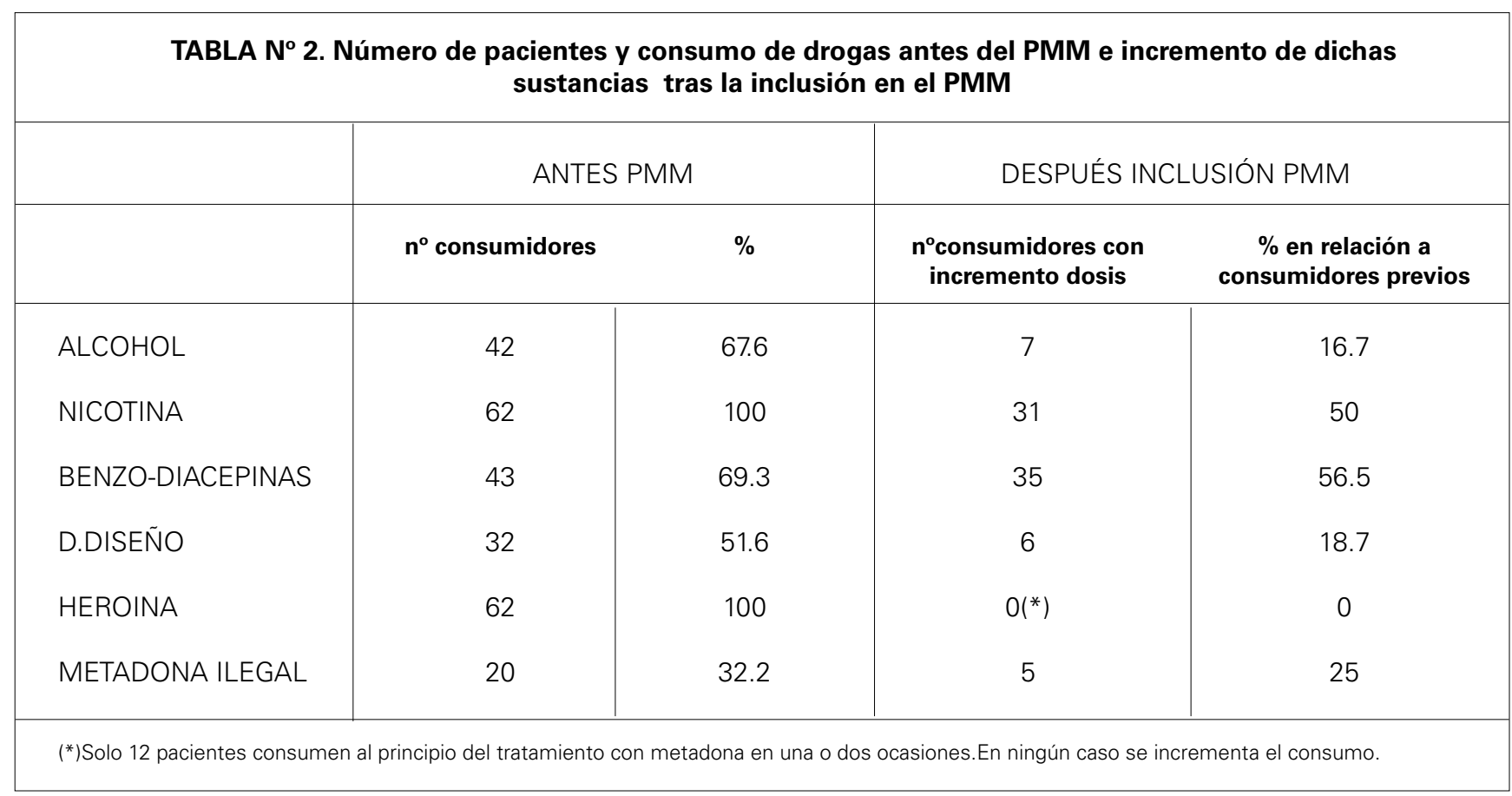

todas las propiedades fisiológicas de la heroína. Se sintetizó en Alemania durante la segunda guerra mundial y se introdujo en el tratamiento de toxicómanos por primera vez en USA en 1940. A principios de los años sesenta comenzaron a estructurarse los Programas de Mantenimiento con Metadona (PMM) y en años sucesivos se iniciaron en otros países como Canadá (1963) Suecia (1966) Reino Unido y Holanda (1968) Australia (1970) Hongkong (1972) Francia (1973) Italia y Suiza (1975). La forma y extensión en el uso de la metadona varia de unos países a otros por limitaciones o regulaciones gubernamentales que van cambiando con el tiempo.

En España la primera normativa relativa a su prescripción, uso y dispensación se creó en 1983 ( O. M. 20 Mayo ) (1). Con posterioridad se dictó la O. M. De 31 Octubre 1985 (2) que regulaba los tratamientos de deshabituación con metadona a dependientes a opiáceos. La Resolución de 22-Noviembre-1985 de la Dirección General de Salud Pública daba normas sobre la dosificación y criterios de aplicación del tratamiento con metadona. El R. D. 75/90 de 19 de Enero de 1990, del Ministerio de Sanidad y Consumo regula- ba los tratamientos en personas dependientes y el R. D. 1131/90 de 14- Noviembre-1990 modificaba el artículo 3.3 del R.D. 75/90, sobre la elaboración, conservación y dispensación de la medicación a las personas en tratamiento. El R.D. 5/96 de 15-Enero-1996 en el apartado 3.1 del R.D. 75/90 adicionaba a la lista de principios activos citados el levo-alfa-acetilmetadol (LAAM) (3).

La evaluación de la eficacia de los PMM según diversos indicadores sociológicos como tasas de empleo o criminalidad y otros indicadores sanitarios como la disminución de la infección por VIH, hepatitis y disminución de consumo de opiáceos aportan datos globalmente positivos (4-8).

El objetivo de este estudio fue constatar la eficacia de un PMM en un centro penitenciario y valorar los cambios sociológicos y sanitarios que experimentan los pacientes en tratamiento, lo que repercute globalmente en su adaptación y en la disminución de riesgos de infección en el medio penitenciario. El segundo objetivo fue considerar la injerencia del consumo de otras drogas contaminantes de los programas y obtener referencias del consumo ilegal de metadona. 
TABLA $N^{\circ} 3$ Frecuencia de consumo de cannabis, cocaina y heroína antes y después de la inclusión en el PMM

\begin{tabular}{|c|c|c|c|c|}
\hline \multicolumn{5}{|l|}{ CANNABIS } \\
\hline & \multicolumn{4}{|c|}{ FRECUENCIA DE CONSUMO } \\
\hline & \multicolumn{2}{|r|}{ ANTES PMM } & \multicolumn{2}{|c|}{ DESPUÉS INCLUSIÓN PMM } \\
\hline & casos & $\%$ en relación a consumidores: 59 & casos & $\%$ en relación a consumidores: 44 \\
\hline Diaria & 29 & 49.1 & 11 & 25 \\
\hline 1 a 6 veces/ semana & 14 & 23.7 & 7 & 15.9 \\
\hline$<1$ semana & 16 & 55.2 & 26 & 59.1 \\
\hline \multicolumn{5}{|l|}{ COCAÍNA } \\
\hline & \multicolumn{4}{|c|}{ FRECUENCIA DE CONSUMO } \\
\hline & \multicolumn{2}{|r|}{ ANTES PMM } & \multicolumn{2}{|c|}{ DESPUÉS INCLUSIÓN PMM } \\
\hline & casos & \% en relación a consumidores: 57 & Casos & \% en relación a consumidores: 27 \\
\hline Diaria & 29 & 50.9 & 14 & 51.8 \\
\hline 1 a 6 veces/ semana & 11 & 19.3 & 7 & 25.9 \\
\hline$<1$ semana & 17 & 29.8 & 6 & 22.2 \\
\hline \multicolumn{5}{|l|}{ HEROÍNA } \\
\hline & \multicolumn{4}{|c|}{ FRECUENCIA DE CONSUMO } \\
\hline & \multicolumn{2}{|r|}{ ANTES PMM } & \multicolumn{2}{|c|}{ DESPUÉS INCLUSIÓN PMM } \\
\hline & casos & \% en relación a consumidores: 62 & casos & $\%$ en relación a consumidores: 12 \\
\hline Diaria & 60 & 96.8 & 0 & 0 \\
\hline 1 a 6 veces/ semana & 2 & 3.2 & 0 & 0 \\
\hline$<1$ semana & 17 & 29.8 & 12 & 100 \\
\hline
\end{tabular}

TABLA №4. Comparación de distintas variables antes y después de la inclusión en el PMM.

\begin{tabular}{|c|c|c|c|c|}
\hline & \multicolumn{2}{|c|}{ ANTES PMM } & \multicolumn{2}{|c|}{ DESPUÉS INCLUSIÓN PMM } \\
\hline & \multicolumn{2}{|c|}{$\mathrm{n}^{0}$ casos } & \multicolumn{2}{|c|}{$\mathrm{n}^{0}$ casos } \\
\hline & $\mathrm{SI}$ & NO & SI & NO \\
\hline DELITOS & 62 & 0 & 24 & 38 \\
\hline INTENTO SUICIDIO & 31 & 31 & 14 & 48 \\
\hline COMPARTIR JERINGUILLAS & 51 & 11 & 10 & 52 \\
\hline PROSTITUCIÓN & 17 & 45 & 9 & 53 \\
\hline DEMANDA Tt ${ }^{\circ}$ PSIQUIÁTRICO & 28 & 34 & 18 & 44 \\
\hline \multirow[t]{2}{*}{ AUTOCONTROL } & 16 & 46 & 58 & 4 \\
\hline & SI & NO & Disminución: Si (*) & Disminución: No(*) \\
\hline AUTOAGRESIVIDAD & 28 & 34 & 19 & 9 \\
\hline HETEROAGRESIVIDAD & 38 & 24 & 32 & 6 \\
\hline
\end{tabular}




\section{PACIENTESY MÉTODOS}

El estudio se llevó a cabo en el centro penitenciario de Brians en Barcelona. Se diseñó un estudio preexperimental con un solo grupo pre-post intervención. Se realizó una entrevista a los pacientes en una única ocasión, y se recogió información retrospectiva sobre el estado de cada sujeto antes y después de recibir terapia con metadona. La muestra fue de 62 internos, 31 mujeres y 31 hombres. La asignación de los sujetos no fué aleatoria por haber pocos pacientes en tratamiento con metadona en el centro lo que dificultaba el muestreo aleatorio. Los pacientes accedieron al estudio de forma voluntaria; se les informó de la intencionalidad del estudio a fin de obtener su consentimiento. Los internos recibían una dosis media de metadona de 81,1 mg ( rango 20-245 mg). Mediante entrevista personal semiestructurada para evitar sesgos de tres facultativos entrevistadores, se aplicó un cuestionario con preguntas cerradas y homogeneidad de ítems que comprendía los datos generales siguientes: edad, estado civil, raza, lugar de nacimiento, nivel de estudios, nivel laboral, procedencia o no de familia estructurada, años de drogadicción y tiempo de inclusión en el programa. Las variables a considerar fueron: relación familiar, trabajo, nivel socioeconómico, autoestima, autocontrol, problemática legal, tratamiento psiquiátrico, intentos de suicidio, hábito de compartir jeringuillas, prostitución, hetero y autoagresividad e historia de drogadicción. En esta última variable se preguntó sobre los consumos de tabaco, alcohol, cannabis, heroína, cocaína, benzodiacepinas y drogas de diseño así como consumo de metadona ilegal.

El autocontrol se definió como la inhibición o control de impulsos por parte del sujeto. La autoagresividad se definió como la producción de lesiones físicas en el mismo sujeto y la heteroagresividad como la agresión y producción de lesiones físicas a otros sujetos. Los intentos de suicidio se definieron como los intentos de autolisis reconocidos, atendidos o no en centros asistenciales. El tratamiento psiquiátrico se definió como demanda específica por parte del afecta- do sin valorar el seguimiento psicológico que reciben los pacientes en PMM.

La evaluación del cuestionario se obtuvo de las respuestas subjetivas de los sujetos encuestados. La relación familiar, trabajo, nivel socioeconómico, se valoraron como mejor, igual o peor situación antes de la terapia con metadona y durante el PMM. La problemática legal, tratamiento psiquiátrico, intentos de suicidio, hábito de compartir jeringuillas, auto- y heteroagresividad y prostitución se valoraron según respuestas positivas o negativas (sí-no, mejor-peor).

El tratamiento estadístico fue aplicación de pruebas no paramétricas, análisis de diseño con datos apareados para variables categóricas, dentro del programa estadístico SPSS para Windows. Cuando las respuestas eran binarias, ( mejor o peor, ) se codificaron en 0 y 1 y se aplicó a cada variable la comparación de dos proporciones: prueba de simetria de Mc Nemar, antes y durante el PMM, procedimiento NPar Test Mc Nemar del sistema SPSS. Cuando las respuestas se categorizaron de forma ordinal en tres niveles ( peor, igual o mejor) se codificaron en 0,1 y 2. Los consumos de cannabis, heroína y cocaína se agruparon en dosis nula, baja, media y alta y se codificaron en $0,1,2$ y 3 . Se aplicó el test de Friedman, dentro del análisis con datos apareados para variables ordinales. No se realizaron pruebas de significación a los consumos de alcohol, drogas de diseño y benzodiacepinas por presentarse dudas en las respuestas de algunos sujetos, en cuanto a cantidades de consumo, que dificultaban su clasificación, ni en las variables auto- $y$ heteroagresividad ya que no toda la muestra presentaba este carácter.

\section{RESULTADOS}

Los resultados se evaluaron en porcentajes con el estadístico frecuencias, ya que la mayoria de las variables consideradas eran categóricas. La edad media de los pacientes valorados fué de 30,3 años (rango 1940). La raza más frecuente fué la blanca representan-

TABLA N 5. Comparación variables antes y después de la inclusión en el PMM: Test Mc Nemar

\begin{tabular}{|c|c|c|}
\hline & Estadístico & valor $p$ \\
\hline Delitos ${ }^{(1)}$ & Binomial exacta & 0.0001 \\
\hline Intento Suicidio (1) & Binomial exacta & 0.0000 \\
\hline Compartir Jeringuillas ${ }^{(1)}$ & Chi-cuadradro: 39.02 & 0.0000 \\
\hline Prostitución (1) & Binomial exacta & 0.0078 \\
\hline Tto Psiquiátrico (1) & Binomial exacta & 0.002 \\
\hline Autocontrol ${ }^{(2)}$ & Chi-cuadrado: 40.02 & 0.0000 \\
\hline
\end{tabular}


TABLA Nº6. Comparación de distintas variables antes y después de la inclusión en el PMM.

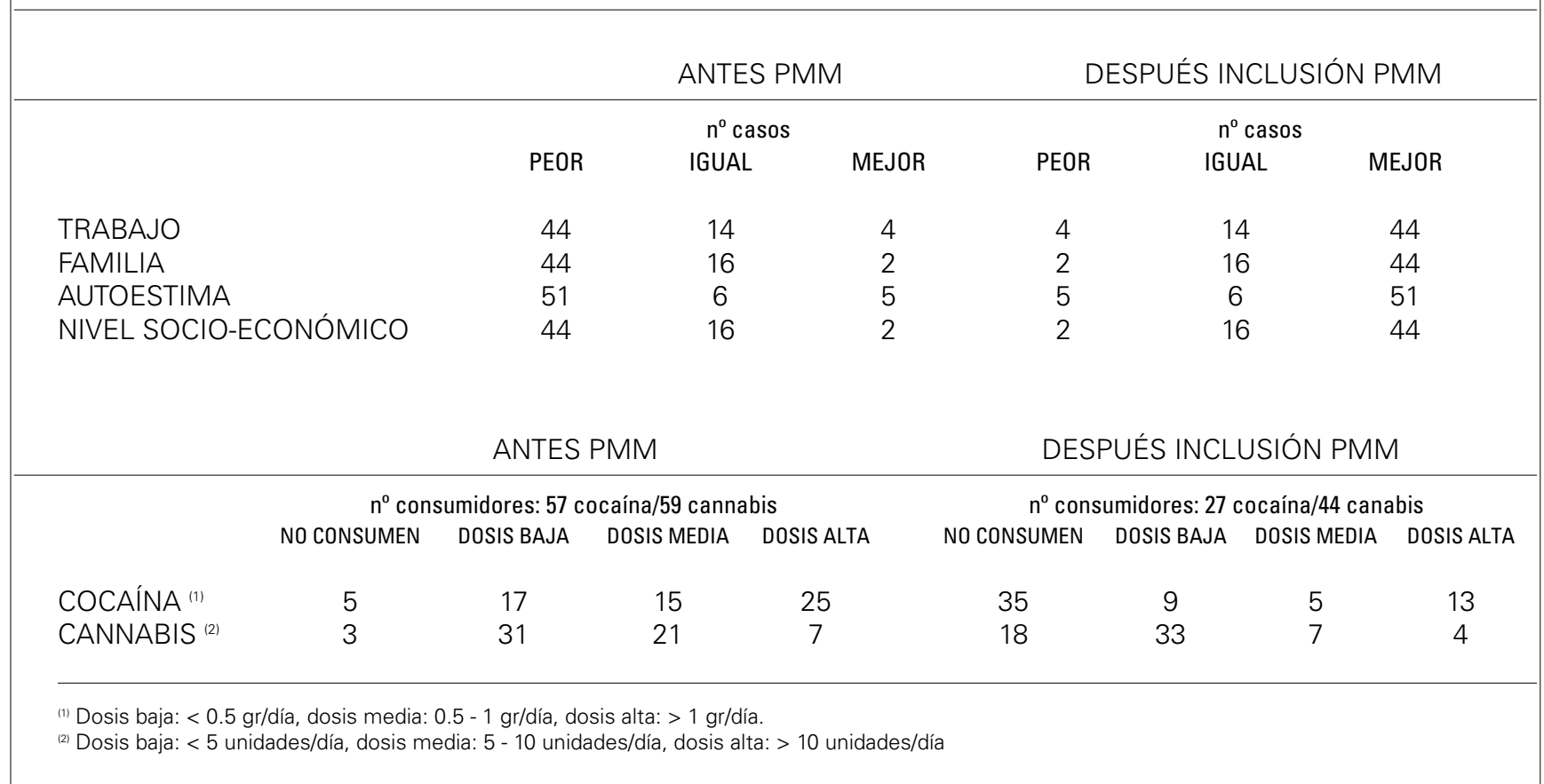

do el 82,3\%( 51 casos). Habían nacido en Cataluña el $67,7 \%$ (42 casos). Estaban solteros el 46,8\% ( 29 casos), convivían con pareja el 19,3\% ( 12 casos), eran casados el 16,1\% ( 10 casos), separados el 8,1\% ( 5 casos)y viudos el 9,7\% ( 6 casos). Habían cursado estudios básicos, completos o incompletos el 59,7\%; ( 37 casos) BUP o FP el 24,2\% (15 casos); eran analfabetos el 14,5\% ( 9 casos)y tenían estudios universitarios el 1,6\% ( 1 caso). En cuanto a la familia, provenían de hogares estructurados el 72,6\%. Tenían familiares toxicómanos el 53,2\% ( 33 casos). En relación al trabajo nunca había trabajado el 11,3\% ( 7 casos), eran obreros no cualificados el 67,8\% ( 42 casos) y desempeñaban otros trabajos el 4,4\% ( 3 casos). El tiempo de inclusión en el PMM de menos de 3 años fué 58,1\%, entre 3 y 6 años 32,2\% y entre 7 y 10 años el 9,7\%. Excepto dos casos los pacientes habían seguido el PMM en situación de libertad.

En relación al HIV, eran Ac HIV + el 69,4\%. El rango de CD4 era de 123-927 y la carga viral fue indetectable en 11 casos. Cumplían criterios de SIDA 5 casos siendo la enfermedad debutante la tuberculosis pulmonar. Estos datos sobre VIH y SIDA no se obtuvieron directamente de los encuestados sino del historial médico existente en el centro.

La edad de inicio del consumo de tóxicos se refleja en la Tabla $n^{\circ} 1$. En relación a la heroína, 39 pacientes ( $62,9 \%$ ) habían iniciado el consumo por vía endovenosa y de forma habitual 55 pacientes ( 88,7\%). La frecuencia de consumo era diaria en 60 casos (96,8\%). Respecto a la dosis consumida, menos de 0,5 gr/día la consumían 3 pacientes, entre 0,5-1 gr/día 19 pacientes y más de 1 gr/día 40 pacientes.
En la muestra estudiada y previo a la inclusión en el PMM consumían heroína el 100/\% de los casos $(62$ pacientes) alcohol el 67,7\% (42 casos) tabaco el $100 \%$ ( 62 casos) cannabis el 95,2\% (59 casos) benzodiacepinas el 69,3\% (43 casos) drogas de diseño el 51,6\% (32 casos) cocaína 91,9\% (57 casos) y metadona ilegal el 32,2\% (20 casos). Durante el PMM la ingesta enólica aumentó un 16,7\% (7 casos) la nicotina un $50 \%$ (31 casos) las benzodiacepinas un 56,5\% (35 casos) y las drogas de diseño un $18,7 \%$ ( 6 casos) según resultados expuestos en las Tabla $n^{\circ} 2$. La disminución de consumo de cannabis, cocaína y heroína se expone en la Tabla $n^{\circ} 3$.

Durante su inclusión en el PMM habían mejorado la relación familiar, nivel socioeconómico, trabajo, autoestima y autocontrol. La autoagresividad presente en 28 de los pacientes, previo a su inclusión en el programa, descendió en 19 de ellos. La heteroagresividad, presente en 38 de los encuestados descendió en 32 de ellos durante el tratamiento. La demanda de asistencia psiquiátrica, los intentos de suicidio, la prostitución, el hábito de compartir jeringuillas, la problemática legal, y el consumo de cocaína , cannabis y heroína disminuyeron de forma significativa, según resultados expuestos en las tablas $n^{\circ} 4$ a 7 .

\section{DISCUSIÓN}

Los resultados del estudio corroboran que los PMM son eficaces al conseguir mejoría evolutiva en indicadores sociológicos como problemática legal y 
TABLA N 7 COMPARACIÓN VARIABLES ANTES Y DESPUÉS DE LA INCLUSIÓN EN EL PMM:TEST DE FRIEDMAN

\begin{tabular}{|c|c|c|c|}
\hline & ESTADÍSTICO & GL & VALOR P \\
\hline Trabajo (1) & Chi-cuadrado: 47.03 & 1 & 0.0000 \\
\hline Familia ${ }^{(1)}$ & Chi-cuadrado: 54.25 & 1 & 0.0000 \\
\hline Autoestima ${ }^{(1)}$ & Chi-cuadrado: 43.61 & 1 & 0.0000 \\
\hline Nivel Socio-económico (1) & Chi-cuadrado: 54.28 & 1 & 0.0000 \\
\hline Consumo Cocaína ${ }^{(2)}$ & Chi-cuadrado: 31.22 & 1 & 0.0000 \\
\hline Consumo Heroína (2) & Chi-cuadrado: 48.02 & 1 & 0.0000 \\
\hline Consumo Cannabis ${ }^{(2)}$ & Chi-cuadrado: 19.75 & 1 & 0.0000 \\
\hline
\end{tabular}

criminalidad, reconocido en varios estudios. La retención en el programa es la clave para que los pacientes obtengan beneficios, los tratamientos cortos son ineficaces debido a que el sistema endorfínico tarda en reequilibrarse (9-13). Algunos autores no admiten los beneficios del programa de forma tan contundente; reconocen que la inclusión en el PMM origina menor consumo de drogas pero que la criminalidad continua (14). El nivel socioeconómico de los pacientes en tratamiento se eleva, especialmente cuando coexiste el soporte psicosocial (15) y se establecen buenas relaciones con los educadores del programa (16). Las relaciones familiares y el acceso a la actividad laboral son más favorables, resultados observados por otros autores (17).

En nuestro estudio los pacientes conseguían tener mayor autocontrol y aumentaba su autoestima, disminuían los intentos de suicidio, y la necesidad de seguir tratamiento psiquiátrico. Estos rasgos de comportamiento o carácter no se relacionaron con diagnósticos psiquiátricos previos, sin embargo, otros estudios establecen que en los pacientes en tratamiento los resultados son independientes de psicopatología comórbida o alteraciones psiquiátricas establecidas (18). Los datos sobre trastornos de personalidad en adictos a drogas, se situan entre el $35-54 \%$ (19). No obstante, estos pacientes no presentan diferencias en cuanto a retención en los programas y pueden cambiar sus hábitos de forma similar al resto del grupo (20).

En nuestro estudio el rango de la dosis de metadona administrada a los pacientes era muy amplio de 20$240 \mathrm{mg}$ ( dosis media 81,1 mg). Esto indica que las dosis deben ser individualizadas y con seguimiento personalizado para conseguir que el sujeto no presente deseos de consumo y según el grado de adicción previo. También influye la farmacocinética de la metadona que puede presentar variabilidad interpersonal. En general se considera que dosis menores de $20 \mathrm{mg}$ de metadona mantienen la permanencia en el programa pero no suprimen el uso ilícito de otras drogas, es decir existe efecto dosis -respuesta (21-22). La partici- pación de los pacientes en la dosis de metadona y días de tratamiento da mejores resultados; la flexibilidad es positiva en cuanto a retención en el programa (23). Otro factor relacionado con la dosis de mantenimiento es el metabolismo acelerado de la metadona. Algunos pacientes presentan este aumento del metabolismo de la droga, en especial si consumen otras drogas que inducen el sistema enzimático afectando a los niveles séricos. En general, concentraciones de $100 \mathrm{ng} / \mathrm{ml}$ se consideran adecuadas para el mantenimiento del drogodependiente. Obviamente los análisis del nivel sérico no son disponibles de forma rutinaria pero en pacientes con dificultades de control su determinación puede ser de utilidad (24).

En la actualidad los tratamientos con metadona en prisiones están bien establecidos en nuestro país.

Las experiencias más antíguas para el tratamiento de drogodependientes en prisión se remontan a 1914, realizada por Lichtensteinen en USA (25). En otros paises como Inglaterra, la implantación del programa demostró que los programas debían seguirse como norma para evitar el hábito de compartir jeringuillas, la infección por VIH, hepatitis y SIDA (26-27-28).

En nuestro estudio se comprueba el alto grado de seguimiento clínico que tienen los pacientes en el centro penitenciario, referente a control de infección por $\mathrm{VIH}$, tratamiento antirretroviral, tuberculosis, hepatitis y patologías asociadas. Los centros penitenciarios son un buen medio para llevar a cabo estudios epidemiológicos de distintos tipos en relación con consumo de drogas y patologías citadas(29-30-31). En la población femenina recluida, el PMM permite la prevención de riesgos en el embarazo y el seguimiento perinatal ( 32).

Referente al segundo objetivo valorar el consumo de otras drogas en los programas PMM los resultados presentan limitaciones o sesgos en cuanto los pacientes se hallaban recluidos en el centro, pero la información se obtuvo en sujetos que estuvieron en el PMM con anterioridad a su ingreso en el centro. En nuestra muestra se comprueba el consumo de otras drogas, con aumento del consumo de alcohol , tabaco, benzo- 
diacepinas y de drogas de diseño. Solamente 12 pacientes indicaron consumo de heroína en una o dos ocasiones durante su inclusión en el PMM. Otros estudios coinciden en que existe un consumo simultáneo con otras drogas en un $20 \%$ de pacientes en tratamiento (33). Una de las drogas que se mezclan con más frecuencia son las benzodiacepinas hecho constatado por nosotros.

En nuestro estudio habían consumido metadona ilegal un 32,2\% de la muestra antes de su inclusión en el programa y en muy pocos casos referían conocer alguna muerte por metadona. Tanto en situación carcelaria como en libertad los PMM se consideran relativamente seguros.

Por el momento, en nuestro país las listas de espera para adherirse a los PMM van en aumento, tanto en situación carcelaria como ambulatoria. Muchos drogodependientes comprenden las ventajas integrales del tratamiento; otros no son partidarios , tienen recelo e incluso pueden presentar un "miedo patológico" a la desintoxicación con metadona. Los resultados del programa son mejores si los pacientes no presentan este recelo o miedo a seguir el tratamiento, según se ha comprobado en varios estudios (34).

Los PMM han implicado siempre problemática burocrática, política y social en los distintos paises (35) pero como profesionales de la salud se han de considerar como una respuesta médica a un problema médico y de salud.

En conclusión el tratamiento con metadona representa una estrategia terapéutica válida tanto en Centros Penitenciarios como de forma ambulatoria. Constituyen una alternativa eficaz para la consecución de objetivos de salud, disminuyendo conductas de riesgo para VIH y otras patologías asociadas y modifican positivamente aspectos sociales y legales.

\section{BIBLIOGRAFÍA}

Ministerio de Sanidad y Consumo. Orden de 23- Mayo-1983 por la que se regulan los tratamientos con metadona ( BOE 28-Mayo-1983).

Ministerio de Sanidad y Consumo. Orden de 31-Octubre1985 por la que se regulan los tratamientos de deshabituación con metadona ( BOE 9-Noviembre-1985).

Ministerio de Sanidad y Consumo. Real Decreto 75/90 de 19-Enero-1990 por el que se regulan los tratamientos con opiáceos de personas dependientes de los mismos (BOE 23-Enero-1990).

Información Terapéutica del Sistema Nacional de Salud. Vol $15 ; n^{\circ} 5,1991$
Bertschy R, Gilles A. Methadone maintenance treatment. An update. European Arch Psychiatry Clin Neurosci 1995; 245 (2): 114-24

Murray JB. Effectiviness of methadone maintenance for heroin addiction. Psychol Rep 1998 Aug; 83 (1): 295-2.

Hermstad R, Johannenssen G, Michelsen K. What effects does methadone maintenance treatment have on the rahabilitation of heroin addicts?( abstract). Tidss Nor Laegeforen 1998 Aug 30; 118 (20): 3139-41.

Hartel D, Schoenbaum E. Methadone treatment protects against HIV infection, two decades of experience in the Bronx, New York City. Public Health Rep 1998 Jun; 113 suppl 1: 107-15.

Farrell M, Wards j, Mattick R. Hall W, Stimson G, Jarlais D et al. Methadone maintenance treatment in opiate dependence. Br med J 1994; 309: 997-1001.

Bell J, Hall W, Byth K. Changes in criminal activity after entering methadone maintenance. Br J Addiction 1992; 87: 251-8.

Rittmannsberger H, Ruschak M, Silberbarer C. Methadon substitutions behandlung. Ergebinisse einer. Evaluation nach 3 pahrighem bestenen in einen osterreichen Bundesland (abstract). Nervenarzt 1994 May; 65 (5): 338-44.

Barcia D, Ruiz E, Onate J, Seigner C, Ramo V. Toxicomanía y delincuencia. Estudio de la delincuencia en heroinómanos tratados con metadona. An Psiquiatr 1991; 7/5: 180-3.

Gerstein D, Lewin L. Treating drug problems. N Engl J Med 1990: 323: 844-8.

Goldstein A, Herrera J. Heroin addicts and methadone treatment in Alburquerque: a 22 year follow-up. Drug Alcohol Dependence 1995 Dec; 40 (2): 139-54.

Deglon J. Clinical aspects and evaluation of methadone sustitution therapy. Therapie 1995 Nov-Dec; 50 (6): 537-42.

Tunis S, Delnachi K, Schwart K, Banys P, Sees K. The relationship of counselor and peer alliance to drug use and HIV risk behaviour in a six month methadone detoxification program. Addict Behaviour 1995; 20/3: 395-405.

Meissner A, BurkhardtD, Donath T, Retter F. Erlahrungen der Munchener methadonabulanzem ( abstract). Psychiatri Prax 1997 Nov; 24 (6): 291-5.

Rutherford M, Cacciola J, Alterman A, Cook T. Social competence in opiate addicted individuals: gender differences relationship to psychiatric diagnoses and treatment responses. Addict Behav 1997; 22/3: 419-25.

Brooner R, Schmidt C, Felch L, Bigelow G. Antisocial behaviour of intravenous drug abusers: implications for antisocial personality disorders. Am J Psychiatry 1992; 149: 482-7.

Darke S, Hall W, Swift V. Prevalence symptoms and correlates of antisocial personality disorders among methadone maintenance clients. Drug Alcohol Depend 1994; 34/3: 253-7.

Guthrie S. Pharmacologic interventions for the treatment of opiod dependence and withdrawal. DICP Ann Pharmacother 1990; 24: 721-34. 
Strain E, Sutzer M, Liebson I, Bigelow G. Dose-response effects of methadone in the treatment of opiod dependence. Ann Intern Med 1993; 119: 23.

Maddux J, Prihodat J, Vogtsberger K. The relationship of methadone dose and other variables to outcomes of methadone maintenance. Am J Addict 1997; 6 (3): 246-55.

Bell J, Seres V, Bowron P, Lewis J, Batey R. The use of serum methadone levels in patients receiving methadone maintenance. Clin Pharmacol Ther 1988; 43: 623-9.

Inciardi J, Martin S. Drug abuse treatment in criminal justice settings. J Drug Issues 1993; 1: 1-6.

Farrell M, Strang J. Drugs, HIV and prison: time for a policy reappaisal. B Med J 1991;302: 1477-8.

Inciardi J, Martin S. Drug abuse treatment in criminal justice settings. J Drug Issues 1993; 1: 1-6.

Novick D, Joseph H, Croxon T, Saltsitz E, Wang G, Rickman B et al.. Absence of antibody to human inmunideficiency virus in long term socially rehabilitated methadone maintenance patients. Arch Intern Med 1990; 150: 97-9.
Magura S, Kang S, Shapiro J, O'Day J. Evaluation of an AIDS education model for women drug users in jail. Int $J$ Addict 1995 Feb; 30 (3): 259-73.

Magura S, Kang S, Shapiro J. Outcomes of intensive AIDS education for male adolescents drug users in jail. J Adolesc Health 1994 Sep; 15 (6): 457-63.

Magura S, Kang S, Shapiro J, O'Day J. HIV risks among women injecting drug users who are in jail. Addiction 1993 Oct; 88 (10): 1351-60.

Legeune C, Ropert J, Montamat S, Flochtudal C, Mazy F, Wijkhnisen N, Froman F. Medicosocial outcome in 59 infants born to drug addicted mothers. J Gynecologic Obstetrique Biolol Reproduction 1997; 26 (4): 395-404.

Sanchéz L. Alcoholismo y drogodependencias. En: Foz M, erill S, Soler-Argilaga C. Terapéutica en Medicina Interna. Ed Doyma. Barcelona 1987: 896.

Mc Lellan A, Woody G. Prevalence and diagnostic reliability of methadone maintenance detoxification fear. Am J Psychiatry 1986 Jun; 143 (6): 739-43.

Newman R. Methadone maintenance treatment definig and evaluation and sucess. N Eng J Med 1987; 317: 447-50. 